\title{
MULTIFRACTIONAL PROCESSES WITH RANDOM EXPONENT
}

\author{
Antoine Ayache and Murad S. Taqqu
}

\begin{abstract}
Multifractional Processes with Random Exponent (MPRE) are obtained by replacing the Hurst parameter of Fractional Brownian Motion (FBM) with a stochastic process. This process need not be independent of the white noise generating the FBM. MPREs can be conveniently represented as random wavelet series. We will use this type of representation to study their Hölder regularity and their self-similarity.
\end{abstract}

\section{Introduction}

Fractional Brownian Motion (FBM) with Hurst parameter $H \in(0,1)$, will be denoted $\left\{B_{H}(t)\right\}_{t \in[0,1]}$. It is well known that, up to a multiplicative constant which only depends on $H, B_{H}(t)$ can be represented through the Wiener integral

$$
B_{H}(t)=\int_{\mathbb{R}}\left((t-x)_{+}^{H-1 / 2}-(-x)_{+}^{H-1 / 2}\right) d W(x),
$$

where $u_{+}=\max (u, 0)$, which is called the moving-average representation of FBM. The measure $W$ is Gaussian and independently scattered. Another well-known integral representation of FBM is its harmonizable representation,

$$
B_{H}(t)=\int_{\mathbb{R}} \frac{e^{i t \xi}-1}{i \xi|\xi|^{H-1 / 2}} d \widehat{W}(\xi) .
$$

2000 Mathematics Subject Classification. Primary: 60G18, 60G17; Secondary: $65 \mathrm{~T} 16$.

Key words. Hölder regularity, fractional Brownian motion, self-similarity, sample path properties.

This research was partially supported by the NSF Grant DMS-0102410 at Boston University. 
The random measures $d W$ and $d \widehat{W}$ satisfy a Parseval type relation, namely for any function $f \in L^{2}(\mathbb{R})$ one has almost surely,

$$
\int_{\mathbb{R}} f(x) d W(x)=\int_{\mathbb{R}} \hat{f}(\xi) d \widehat{W}(\xi),
$$

where

$$
\hat{f}(\xi)=\int_{\mathbb{R}} e^{-i \xi \cdot x} f(x) d x
$$

is the Fourier transform of $f$. The complex-valued random measure $d \widehat{W}$ can therefore be viewed as the Fourier transform of the real-valued random measure $d W$. Observe that $d \widehat{W}$ is completely determined by the Relation (1.3).

FBM was introduced in 1940 by Kolmogorov as a way to generate Gaussian "spirals" in Hilbert space [9] and it was made popular by Mandelbrot and Van Ness [12] in 1968. This process has turned out to be very useful in both theory and applications. It has been used to model phenomena in hydrology, economics, finance, physics and telecommunications. For example, Leland, Taqqu, Willinger and Wilson have provided experimental evidence that computer traffic data exhibit long range dependence $[\mathbf{1 0}]$ and since then FBM has been applied with some success as a model in telecommunications (see for instance [15]). The monograph of Doukhan, Oppenheim and Taqqu [6] offers a systematic treatment of FBM, as well as an overview of different areas of applications.

One of the main interests of the FBM in modeling is that its Hölder regularity can be prescribed via its Hurst parameter. Actually, $\alpha_{F B M}(t)$, the pointwise Hölder exponent of the FBM at any point $t$, satisfies almost surely

$$
\alpha_{F B M}(t, \omega)=H,
$$

and $\beta_{F B M}(J)$, its uniform Hölder exponent over an arbitrary non-degenerate interval $J$, satisfies almost surely

$$
\beta_{F B M}(J, \omega)=H .
$$

The higher the Hölder exponents, the smoother the process.

The exponents $\alpha$ and $\beta$ are defined as follows. Let $\{X(t)\}_{t \in T}$ be a random field with continuous and nowhere differentiable trajectories, defined over a rectangle $T$ of $\mathbb{R}^{d}$ (that is a set of the form $\prod_{k=1}^{d}\left[\gamma_{k}, \delta_{k}\right]$ ). The local Hölder regularity of $\{X(t)\}_{t \in T}$ in a neighbourhood of each point $t$ can be measured through its pointwise Hölder exponent, namely 
the stochastic process $\left\{\alpha_{X}(t)\right\}_{t \in T}$ defined for every $t$ and $\omega$ as,

$$
\alpha_{X}(t, \omega)=\sup \left\{\alpha, \limsup _{h \rightarrow 0} \frac{|X(t+h, \omega)-X(t, \omega)|}{|h|^{\alpha}}=0\right\} .
$$

The global Hölder regularity of $\{X(t)\}_{t \in T}$ over a non-degenerate rectangle $J \subset T$ can be measured through its uniform Hölder exponent over $J$, namely the random variable $\beta_{X}(J)$ defined for every $\omega$ as

$$
\beta_{X}(J, \omega)=\sup \left\{\beta, \sup _{s, s^{\prime} \in J} \frac{\left|X(s, \omega)-X\left(s^{\prime}, \omega\right)\right|}{\left|s-s^{\prime}\right|^{\beta}}<\infty\right\} .
$$

Observe that one always has

$$
\beta_{X}(J, \omega) \leq \inf _{t \in J} \alpha_{X}(t, \omega) .
$$

The pointwise and uniform Hölder exponents of the FBM are constant, since they do not depend on the location (i.e. the point $t$ or the interval $J$ ) nor $\omega$. This may be undesirable in some situations. For example, FBM is not well adapted to the modeling of non-homogenous materials. Or consider the field of image synthesis: FBM has been frequently used for generating artificial mountains [4], but one obtains in this way mountains whose irregularity is the same everywhere. This is not realistic, since it does not take into account erosion or other meteorological phenomena which smooth some parts of the mountains more than others. The Multifractional Brownian Motion (MBM) has been introduced, independently in [16] and [5], to overcome such limitations of the FBM. Recall that this Gaussian process can be obtained by substituting to the Hurst parameter of the FBM, a function $H(\cdot)$ with values in an arbitrary compact interval $[a, b] \subset(0,1)$. Because of the Relation (1.3) this substitution can be made in the integrals (1.1) or (1.2). Thus the $\operatorname{MBM}\left\{B_{H(t)}(t)\right\}_{t \in[0,1]}$ has the following integral representations:

$$
B_{H(t)}(t)=\int_{\mathbb{R}}\left((t-s)_{+}^{H(t)-1 / 2}-(-s)_{+}^{H(t)-1 / 2}\right) d W(s)
$$

and

$$
B_{H(t)}(t)=\int_{\mathbb{R}} \frac{e^{i t \xi}-1}{i \xi|\xi|^{H(t)-1 / 2}} d \widehat{W}(\xi) .
$$

As in the case of FBM, these representations are respectively called the moving average and the harmonizable representation of the MBM. It has been shown in $[\mathbf{1 6}]$ and in $[\mathbf{5}]$, that when $\beta_{H}([0,1])$, the uniform Hölder exponent over the interval $[0,1]$ of the function $H(\cdot)$, satisfies the 
condition,

$$
\sup _{t \in[0,1]} H(t)<\beta_{H}([0,1]),
$$

then the Hölder regularity of the MBM can be prescribed via $H(\cdot)$. Namely, the pointwise Hölder exponent of the MBM at any point $t$, satisfies almost surely,

$$
\alpha_{M B M}(t, \omega)=H(t),
$$

and the uniform Hölder exponent of the MBM over any non-degenerate interval $J \subset[0,1]$ verifies almost surely,

$$
\beta_{M B M}(J, \omega)=\inf _{t \in J} H(t) .
$$

Papanicolaou and Sølna [14] have observed that the deterministic functional parameter $H(\cdot)$ of the MBM can be replaced by a stochastic process $\{S(t)\}_{t \in[0,1]}$. They suppose, for example, that $\{S(t)\}_{t \in[0,1]}$ is a stationary process with smooth paths and decaying correlation function that is independent on the white noise, i.e. the Wiener process $\{W(x)\}_{x \in \mathbb{R}}$ which appears for example in Relation (1.1) (see [14, Subsection 4.1, p. 484]). We will call such extensions of the MBM, Multifractional Processes with Random Exponent (MPRE). To define an MPRE we need the following ingredients:

- $\left\{B_{H}(t)\right\}_{(t, H) \in[0,1] \times[a, b]}$, a Gaussian field with integral representations (1.1) and (1.2). Contrarily to FBM this field depends both on $t$ and $H$. It is defined over $[0,1] \times[a, b]$ where $[a, b] \subset(0,1)$ is an arbitrary fixed compact interval.

- $\{S(t)\}_{t \in[0,1]}$, a stochastic process with values in $[a, b]$.

Convention. We suppose throughout this paper that $0<a<b<1$.

Definition 1.1. The Multifractional Process with Random Exponent (MPRE) with parameter $\{S(t)\}_{t \in[0,1]}$ is the stochastic process $\{Z(t)\}_{t \in[0,1]}$ defined as follows: any trajectory $t \mapsto Z(t, \omega)$ is the composition of the function $f_{1}:[0,1] \rightarrow[0,1] \times[a, b], t \mapsto(t, S(t, \omega))$ and $f_{2}:[0,1] \times[a, b] \rightarrow$ $\mathbb{R},(t, H) \mapsto B_{H}(t, \omega)$. Thus, for any $t \in[0,1]$ and $\omega$, one has

$$
Z(t, \omega)=f_{2}\left(f_{1}(t)\right)=B_{S(t, \omega)}(t, \omega) .
$$

Observe that we do not necessarily suppose that the stochastic parameter $\{S(t)\}_{t \in[0,1]}$ in the MPRE is independent of the white noise $W$ in (1.10), nor that it is stationary. When $\{S(t)\}_{t \in[0,1]}$ is independent on the white noise $W$, the main results on MBM can be extended readily to the MPRE. The more general case, where it can be dependent on the white noise, is more tricky. Actually, in that case, for any non-zero 
fixed $t$, the process $\left\{(t-s)_{+}^{S(t, \omega)-1 / 2}-(-s)_{+}^{S(t, \omega)-1 / 2}\right\}_{s \in \mathbb{R}}$ (which depends on the variable $s$ ) is no longer adapted to the natural filtration of $\{W(s)\}_{s \in \mathbb{R}}$ and the MPRE cannot therefore be represented as a usual Itô integral. On the other hand, it is possible to adopt the approach of Definition 1.1 because $S(t, \omega)$ does not involve the variable $s$. By using the series representation (2.3) for $B_{H}(t)$, we will, in fact, be working only with sums.

The paper is organized as follows. In Section 2, we introduce a wavelet decomposition of the field $\left\{B_{H}(t)\right\}_{(t, H) \in[0,1] \times[a, b]}$ and give some properties of this field that will simplify the study of MPREs. Using this wavelet decomposition, we show in Section 3, that the pointwise and uniform Hölder exponents of the MPRE can be prescribed by its random parameter $\{S(t)\}_{t \in[0,1]}$. Finally, in Section 4, we give sufficient conditions for the MPRE to be self-similar (in the sense of marginal distributions) or have stationary increments.

A word about notation. We will be using non-random as well as random constants. To ease the distinction, we use small letters (e.g. $c$ ) to denote non-random constants and capital letters (e.g. $C=C(\omega)$ ) to denote random constants. Moreover, for the sake of simplicity, the stochastic processes considered here are real-valued and often defined on the interval $[0,1]$. Our results remain true when the interval $[0,1]$ is replaced by a compact cube of $\mathbb{R}^{d}$.

\section{Some useful properties of the random field $\left\{B_{H}(t)\right\}_{(t, H) \in[0,1] \times[a, b]}$}

We obtain, in this section, some properties of the field $\left\{B_{H}(t)\right\}_{(t, H) \in[0,1] \times[a, b]}$ that will simplify the study of the MPRE $\{Z(t)\}_{t \in[0,1]}$. Recall that $\left\{B_{H}(t)\right\}_{(t, H) \in[0,1] \times[a, b]}$ and $\{Z(t)\}_{t \in[0,1]}$ are related through Relation (1.15).

We first provide a wavelet decomposition of the field $\left\{B_{H}(t)\right\}_{(t, H) \in[0,1] \times[a, b]}$. Let $\left\{\psi_{j, k}(x)\right\}_{(j, k) \in \mathbb{Z}^{2}}$ be a Lemarié-Meyer wavelet basis of the Hilbert space $L^{2}(\mathbb{R})$. Recall that such basis has the following properties.

$\left(P_{1}\right)$ The functions $\psi_{j, k}$ are generated by dilations and translations of a unique function $\psi$ called a mother wavelet. Namely, for every $j \in \mathbb{Z}$, $k \in \mathbb{Z}$ and $x \in \mathbb{R}$, one has

$$
\psi_{j, k}(x)=2^{j / 2} \psi\left(2^{j} x-k\right),
$$


or equivalently $\widehat{\psi}_{j, k}$, the Fourier transform of $\psi_{j, k}$, satisfies for every $\xi \in \mathbb{R}$,

$$
\widehat{\psi}_{j, k}(\xi)=2^{-j / 2} e^{-i k 2^{-j} \xi} \widehat{\psi}\left(2^{-j} \xi\right) .
$$

In addition, the functions $\psi_{j, k}$, and consequently the functions $\widehat{\psi}_{j, k}$, belong to the Schwartz class $S(\mathbb{R})$. Recall that $S(\mathbb{R})$ is the space of all infinitely differentiable functions $u$ whose derivatives $u^{(n)}$ of any order $n \geq 0$ satisfy for all integer $m$,

$$
\lim _{|t| \rightarrow \infty} t^{m} u^{(n)}(t)=0
$$

Observe that the tails of $u^{(n)}$ decrease faster than any polynomial.

$\left(P_{2}\right)$ For any integers $j$ and $k$ the support of $\widehat{\psi}_{j, k}$ is contained in the domain $\left\{\xi \in \mathbb{R}, \frac{2^{j+1} \pi}{3} \leq|\xi| \leq \frac{2^{j+3} \pi}{3}\right\}$.

$\left(P_{3}\right)$ Up to a multiplicative factor $1 / \sqrt{2 \pi}$ that we will neglect, $\left\{\widehat{\psi}_{j, k}(-\xi)\right\}_{(j, k) \in \mathbb{Z}^{2}}$ forms an orthonormal basis of $L^{2}(\mathbb{R})$.

To obtain a random wavelets series representation of the field $\left\{B_{H}(t)\right\}_{(t, H) \in[0,1] \times[a, b]}$, one fixes $(t, H) \in[0,1] \times[a, b]$, and decomposes its kernel, namely the function $\widehat{f}: \xi \mapsto \frac{e^{i t \xi}-1}{i \xi|\xi|^{H-1 / 2}}$ in the basis $\left\{\widehat{\psi}_{j, k}(-\xi)\right\}_{j, k \in \mathbb{Z}}$. Then one applies the integral $\int_{\mathbb{R}} \widehat{f}(\cdot) d \widehat{W}$ to this decomposition. Since, this integral is an isometry from the Hilbert space $L^{2}(\mathbb{R})$ into the Hilbert space $L^{2}(\Omega)$ of the square integrable and mean-zero random variables, one obtains, in view of $(1.2)$, that for every $(t, H) \in[0,1] \times[a, b]$,

$$
B_{H}(t)=\sum_{j \in \mathbb{Z}} \sum_{k \in \mathbb{Z}} a_{j, k}(t, H) \epsilon_{j, k},
$$

where $\left\{\epsilon_{j, k}\right\}_{j, k \in \mathbb{Z}}$ is a sequence of $\mathcal{N}(0,1)$ Gaussian random variables and where the non-random coefficients $a_{j, k}(t, H)$ are given by

$$
a_{j, k}(t, H)=\int_{\mathbb{R}} \frac{e^{i t \xi}-1}{i \xi|\xi|^{H-1 / 2}} \widehat{\psi}_{j, k}(\xi) d \xi .
$$

We will show later that the series (2.3) is, with probability 1 , uniformly convergent in $(t, H)$. If we define, for every $(x, H) \in \mathbb{R} \times[a, b]$, the function

$$
\Psi(x, H)=\int_{\mathbb{R}} e^{i x \xi} \frac{\widehat{\psi}(\xi)}{i \xi|\xi|^{H-1 / 2}} d \xi,
$$

then by setting $\eta=2^{-j} \xi$ in the integral (2.4) and using (2.2), one gets

$$
a_{j, k}(t, H)=2^{-j H}\left(\Psi\left(2^{j} t-k, H\right)-\Psi(-k, H)\right) .
$$


Observe that the integral (2.5) converges since $\widehat{\psi}$ belongs to $S(\mathbb{R})$ and vanishes in a neighbourhood of the origin. We now give some useful properties of the function $\Psi$.

Lemma 2.1. $\Psi$ is a $C^{\infty}$ function over $\mathbb{R} \times[a, b]$ and its partial derivatives of any order are localized in the variable $x$ uniformly in the variable $H$. As a consequence, for all integers $m$ and $n$ there is a constant $c>0$ (that only depends on $m, n, a$ and $b$ ) such that for every $(x, H) \in \mathbb{R} \times[a, b]$ one has,

$$
\left|\frac{\partial^{m+n}}{(\partial x)^{m}(\partial H)^{n}} \Psi(x, H)\right| \leq c(2+|x|)^{-2} .
$$

Proof: First we will suppose that $x \geq 0$. Let $K$ be the integrand in (2.5), namely the function defined for any $(x, H, \xi) \in \mathbb{R}_{+} \times[a, b] \times \mathbb{R}$, as $K(x, H, \xi)=e^{i x \xi} \frac{\widehat{\psi}(\xi)}{i \xi|\xi|^{H-1 / 2}} . \quad K$ is an infinitely differentiable function in $(x, H)$, whose partial derivatives of any order are bounded uniformly in $(x, H)$ by a $\xi$ integrable function. It follows that for every integers $m$, $n$ and $(x, H) \in \mathbb{R}_{+} \times[a, b]$, one has

$$
\frac{\partial^{m+n}}{(\partial x)^{m}(\partial H)^{n}} \Psi(x, H)=\int_{\mathbb{R}} \frac{\partial^{m+n}}{(\partial x)^{m}(\partial H)^{n}} K(x, H, \xi) d \xi,
$$

which implies that

$$
\left|\frac{\partial^{m+n}}{(\partial x)^{m}(\partial H)^{n}} \Psi(x, H)\right|=\left|\int_{\mathbb{R}} e^{i(2+x) \xi} \frac{\widehat{\phi}(\xi)}{i \xi|\xi|^{H-1 / 2}} d \xi\right|,
$$

where $\widehat{\phi}$ is the function of $S(\mathbb{R})$ defined for every real $\xi$ by $\widehat{\phi}(\xi)=$ $e^{-i 2 \xi} \xi^{m}(\log |\xi|)^{n} \widehat{\psi}(\xi)$. Then integrating twice by parts, one obtains that

$$
\begin{aligned}
\left|\int_{\mathbb{R}} e^{i(2+x) \xi} \frac{\widehat{\phi}(\xi)}{i \xi|\xi|^{H-1 / 2}} d \xi\right| \leq(2+x)^{-2} \int_{\mathbb{R}}\left(\frac{\left|\widehat{\phi}^{\prime \prime}(\xi)\right|}{|\xi|^{H+1 / 2}}+(2 H+1) \frac{\left|\widehat{\phi}^{\prime}(\xi)\right|}{|\xi|^{H+3 / 2}}\right. \\
\left.\quad+(H+1 / 2)(H+3 / 2) \frac{|\widehat{\phi}(\xi)|}{|\xi|^{H+5 / 2}}\right) d \xi \\
\leq c(2+x)^{-2},
\end{aligned}
$$

where the constant $c=5\left(\int_{\mathbb{R}}\left(\sum_{p=0}^{2}\left|\widehat{\phi}^{(2-p)}(\xi)\right|\left(\frac{1}{|\xi|^{a+1 / 2+p}}+\frac{1}{|\xi|^{b+1 / 2+p}}\right)\right)\right) d \xi$. The case where $x<0$ can be treated similarly. 
Let $\left\{\dot{B}_{H}(t)\right\}_{(t, H) \in \mathbb{R} \times[a, b]}$ and $\left\{\ddot{B}_{H}(t)\right\}_{(t, H) \in \mathbb{R} \times[a, b]}$ be respectively the low frequency and the high frequency components of the wavelet representation of $\left\{B_{H}(t)\right\}_{(t, H) \in \mathbb{R} \times[a, b]}$. These fields are defined for every $(t, H) \in \mathbb{R} \times[a, b]$ as,

$$
\begin{aligned}
\dot{B}_{H}(t) & =\sum_{j=-\infty}^{-1} \sum_{k \in \mathbb{Z}} 2^{-j H} \epsilon_{j, k}\left(\Psi\left(2^{j} t-k, H\right)-\Psi(-k, H)\right) \\
& =\sum_{j=1}^{\infty} \sum_{k \in \mathbb{Z}} 2^{j H} \epsilon_{-j, k}\left(\Psi\left(2^{-j} t-k, H\right)-\Psi(-k, H)\right),
\end{aligned}
$$

and

$$
\ddot{B}_{H}(t)=\sum_{j=0}^{\infty} \sum_{k \in \mathbb{Z}} 2^{-j H} \epsilon_{j, k}\left(\Psi\left(2^{j} t-k, H\right)-\Psi(-k, H)\right) .
$$

Clearly, one has

$$
B_{H}(t)=\dot{B}_{H}(t)+\ddot{B}_{H}(t) .
$$

We now provide some properties of $\left\{\dot{B}_{H}(t)\right\}_{(t, H) \in \mathbb{R} \times[a, b]}$ and $\left\{\ddot{B}_{H}(t)\right\}_{(t, H) \in \mathbb{R} \times[a, b]}$. The proofs of the following propositions will be given at the end of this section.

Proposition 2.1. The trajectories of the field $\left\{\dot{B}_{H}(t)\right\}_{(t, H) \in \mathbb{R} \times[a, b]}$ are with probability $1, C^{\infty}$ functions over $\mathbb{R} \times[a, b]$.

Proposition 2.2. There is an event $\Omega_{1}^{*}$ of probability 1 , satisfying the following properties:

(a) For any $\omega \in \Omega_{1}^{*}$, the function $(t, H) \mapsto \ddot{B}_{H}(t, \omega)$ is continuous over $[0,1] \times[a, b]$.

(b) For all $\omega \in \Omega_{1}^{*}$ and all reals $m$ and $M$ such that $a \leq m \leq M \leq b$, the uniform Hölder exponent of the function $(t, H) \mapsto \ddot{B}_{H}(t, \omega)$ over the rectangle $[0,1] \times[m, M]$ is equal to $m$ (see Relation (1.8) for the definition of this exponent). This means that for any arbitrarily small $\epsilon>0$, there is a random variable $C_{1}>0$ (which only depends on $m, M$ and $\epsilon$ ) such that the inequality

$$
\left|\ddot{B}_{H^{\prime}}\left(t^{\prime}, \omega\right)-\ddot{B}_{H^{\prime \prime}}\left(t^{\prime \prime}, \omega\right)\right| \leq C_{1}(\omega)\left(\left|t^{\prime}-t^{\prime \prime}\right|+\left|H^{\prime}-H^{\prime \prime}\right|\right)^{m-\epsilon},
$$

holds for all $\omega \in \Omega_{1}^{*},\left(t^{\prime}, H^{\prime}\right) \in[0,1] \times[m, M]$ and $\left(t^{\prime \prime}, H^{\prime \prime}\right) \in$ $[0,1] \times[m, M]$. 
(c) For all $\omega \in \Omega_{1}^{*}$, the function $H \mapsto \ddot{B}_{H}(t, \omega)$ is Lipschitz over $[a, b]$ uniformly in $t \in[0,1]$. More precisely, there is a random variable $C_{2}>0$ (which only depends on a and $b$ ) such that for all $H^{\prime} \in$ $[a, b]$ and $H^{\prime \prime} \in[a, b]$ one has

$$
\sup _{t \in[0,1]}\left|\ddot{B}_{H^{\prime}}(t, \omega)-\ddot{B}_{H^{\prime \prime}}(t, \omega)\right| \leq C_{2}(\omega)\left|H^{\prime}-H^{\prime \prime}\right| .
$$

The following theorem is a straightforward consequence of Propositions 2.1 and 2.2 .

Theorem 2.1. Proposition 2.2 remains true when the high-frequency field $\left\{\ddot{B}_{H}(t)\right\}_{(t, H) \in[0,1] \times[a, b]}$ is replaced by the field $\left\{B_{H}(t)\right\}_{(t, H) \in[0,1] \times[a, b]}$.

We now state two lemmas that we need in the proofs of Propositions 2.1 and 2.2. The first lemma follows from the Borel-Cantelli lemma and one may refer to $[\mathbf{1 3}]$ or $[\mathbf{3}]$ for example for its proof. The second lemma is a reformulation of a strong version of Kolmogorov-Centsǒv criterion (see Chapter 2 of $[\mathbf{8}]$ ). The proof of that lemma can be found in e.g. [2].

Lemma 2.2. There is a random variable $C_{3}>0$ with finite moments of any order and there is an event $\Omega_{3}^{*}$ of probability 1 , such that for any $\omega \in \Omega_{3}^{*}, j \in \mathbb{Z}$ and $k \in \mathbb{Z}$ one has

$$
\left|\epsilon_{j, k}(\omega)\right| \leq C_{3}(\omega) \sqrt{\log (2+|j|)} \sqrt{\log (2+|k|)} .
$$

Lemma 2.3. Let $\{X(\tau)\}_{\tau \in T}$ be a Gaussian field with continuous trajectories defined on a rectangle $T$ of $\mathbb{R}^{d}$ (i.e. a set of the form $\prod_{k=1}^{d}\left[\gamma_{k}, \delta_{k}\right]$ ). Suppose that for some constants $\mu \in(0,1)$ and $c_{4}>0$, the inequality

$$
\mathbb{E}\left(\left|X\left(\tau^{\prime}\right)-X\left(\tau^{\prime \prime}\right)\right|^{2}\right) \leq c_{4}\left|\tau^{\prime}-\tau^{\prime \prime}\right|^{2 \mu},
$$

holds for every $\tau^{\prime}, \tau^{\prime \prime} \in T$, where $|\cdot|$ is an arbitrary norm on $\mathbb{R}^{d}$. Then, the uniform Hölder exponent over $T$ of the field $\{X(\tau)\}_{\tau \in T}$ is almost surely greater than $\mu$. This means that there is $\Omega_{4}^{*}$, an event of probability 1 , that only depends on $T$, such that for every $\omega \in \Omega_{4}^{*}$ and every arbitrarily small real $\epsilon>0$, the inequality

$$
\left|X\left(\tau^{\prime}, \omega\right)-X\left(\tau^{\prime \prime}, \omega\right)\right| \leq C_{4}(\omega)\left|\tau^{\prime}-\tau^{\prime \prime}\right|^{\mu-\epsilon},
$$

holds for all $\tau^{\prime} \in T$ and $\tau^{\prime \prime} \in T$ (observe that the random variable $C_{4}$ only depends on $\epsilon$ ).

Proof of Proposition 2.1: In view of Lemma 2.1, it is sufficient to show that the series (2.8) and all the series obtained by differentiating it term by term are, with probability 1 , uniformly convergent in the variable $(t, H)$ on each compact set of the form $[-d, d] \times[a, b]$ where the 
real $d>0$ is arbitrary. From now on we will suppose that $\omega \in \Omega_{3}^{*}$ (the probability 1 event introduced in Lemma 2.2) and to simplify our notations we will set $d=1$ without loss of generality. Let us first study the series

$$
\begin{aligned}
A_{n}(t, H, \omega)= & \sum_{j=1}^{\infty} \sum_{k \in \mathbb{Z}} 2^{j H} \epsilon_{-j, k} \\
& \times\left(\frac{\partial^{n}}{(\partial H)^{n}} \Psi\left(2^{-j} t-k, H\right)-\frac{\partial^{n}}{(\partial H)^{n}} \Psi(-k, H)\right),
\end{aligned}
$$

where the integer $n \geq 0$ is arbitrary. Applying the Mean Value Theorem one gets that for any integer $j \geq 1, k \in \mathbb{Z}$ and $(t, H) \in[-1,1] \times[a, b]$ there is a real $\nu \in(-1,1)$ (depending on $j, k$ and $(t, H))$ such that

$$
\begin{aligned}
& \frac{\partial^{n}}{(\partial H)^{n}} \Psi\left(2^{-j} t-k, H\right)-\frac{\partial^{n}}{(\partial H)^{n}} \Psi(-k, H) \\
& =2^{-j} t \frac{\partial^{n+1}}{(\partial x)(\partial H)^{n}} \Psi(\nu-k, H) .
\end{aligned}
$$

Then it follows from Relations (2.7), (2.13), (2.15) and (2.16) that for every $(t, H) \in[-1,1] \times[a, b]$, one has

$$
\left|A_{n}(t, H, \omega)\right| \leq c \sum_{j=1}^{\infty} \sum_{k \in \mathbb{Z}} \frac{2^{-j(1-H)} \sqrt{\log (2+j)} \sqrt{\log (2+|k|)}}{(1+|k|)^{2}}<\infty,
$$

where $c>0$ is a constant that does not depend on $(t, H)$. This proves the uniform convergence of the series (2.15). Using a similar method one can show that for any integer $m \geq 1$ and $n \geq 0$, the series

$$
\sum_{j=1}^{\infty} \sum_{k \in \mathbb{Z}} 2^{-j(m-H)} \epsilon_{-j, k}(\omega) \frac{\partial^{m+n}}{(\partial x)^{m}(\partial H)^{n}} \Psi\left(2^{-j} t-k, H\right)
$$

is uniformly convergent.

Proof of Proposition 2.2: Let us first prove (a) and (c). Using techniques similar to those of Section 3 of $[\mathbf{3}]$, it follows from the inequalities (2.7) and (2.13) that for all $\omega \in \Omega_{3}^{*}$ (the probability 1 event introduced in Lemma 2.2) and any $n \in\{0,1\}$ the series

$$
D_{n}(t, H, \omega)=\sum_{j=0}^{\infty} \sum_{k \in \mathbb{Z}} 2^{-j H} \epsilon_{j, k}(\omega) \frac{\partial^{n}}{(\partial H)^{n}} \Psi\left(2^{j} t-k, H\right),
$$

is uniformly convergent in $(t, H) \in[0,1] \times[a, b]$. Then as the general term of this series is a continuous function in $(t, H)$, it follows that the 
sum of this series, $(t, H) \mapsto D_{n}(t, H, \omega)$ is itself a continuous function over $[0,1] \times[a, b]$. Thus proves Part (a).

Part (c) can be obtained as follows. Applying the Mean Value Theorem one gets that, for any $t \in[0,1], H^{\prime}, H^{\prime \prime} \in[a, b]$, there is a real $\nu \in(a, b)$ such that

$$
\ddot{B}_{H^{\prime}}(t, \omega)-\ddot{B}_{H^{\prime \prime}}(t, \omega)=\left(\frac{\partial}{\partial H}\right) \ddot{B}_{\nu}(t, \omega)\left(H^{\prime}-H^{\prime \prime}\right)
$$

and this inequality implies that

$$
\left|\ddot{B}_{H^{\prime}}(t, \omega)-\ddot{B}_{H^{\prime \prime}}(t, \omega)\right| \leq C_{2}(\omega)\left|H^{\prime}-H^{\prime \prime}\right|
$$

where the random variable $C_{2}$ is defined for every $\omega$ as

$$
C_{2}(\omega)=\sup _{(t, H) \in[0,1] \times[a, b]}\left|\left(\frac{\partial}{\partial H}\right) \ddot{B}_{H}(t, \omega)\right|=\sup _{(t, H) \in[0,1] \times[a, b]}\left|D_{1}(t, H, \omega)\right| .
$$

Observe that this random variable is almost surely finite because of the continuity of the function $(t, H) \mapsto D_{1}(t, H, \omega)$ for almost all $\omega$.

Let us now prove Part (b). It follows from Relations (2.8), (2.9), (2.10), (1.2) and from the formula $(u+v)^{2} \leq 2\left(u^{2}+v^{2}\right)$ that for any $\left(t^{\prime}, H^{\prime}\right) \in[0,1] \times[m, M]$ and $\left(t^{\prime \prime}, H^{\prime \prime}\right) \in[0,1] \times[m, M]$ one has

$$
\begin{aligned}
\mathbb{E}\left(\left|\ddot{B}_{H^{\prime}}\left(t^{\prime}\right)-\ddot{B}_{H^{\prime \prime}}\left(t^{\prime \prime}\right)\right|^{2}\right) \leq & \mathbb{E}\left(\left|B_{H^{\prime}}\left(t^{\prime}\right)-B_{H^{\prime \prime}}\left(t^{\prime \prime}\right)\right|^{2}\right) \\
\leq & \int_{\mathbb{R}}\left|\frac{e^{i t^{\prime} \xi}-1}{|\xi|^{H^{\prime}+1 / 2}}-\frac{e^{i t^{\prime \prime} \xi}-1}{|\xi|^{H^{\prime \prime}+1 / 2}}\right|^{2} d \xi \\
\leq & 2 \int_{\mathbb{R}} \frac{\left|e^{i\left(t^{\prime}-t^{\prime \prime}\right) \xi}-1\right|^{2}}{|\xi|^{2 H^{\prime}+1}} d \xi \\
& +2 \int_{\mathbb{R}} \frac{\left|e^{i t^{\prime \prime} \xi}-1\right|^{2}}{|\xi|}\left(\frac{1}{|\xi|^{H^{\prime}}}-\frac{1}{|\xi|^{H^{\prime \prime}}}\right)^{2} d \xi .
\end{aligned}
$$

We shall now provide an upper bound of each integral in this last inequality. There is no restriction to suppose that $t^{\prime} \neq t^{\prime \prime}$. Setting $\eta=\left(t^{\prime}-t^{\prime \prime}\right) \xi$ in the first integral and using the inequalities $m \leq H^{\prime} \leq M$, one obtains that

$$
\begin{aligned}
\int_{\mathbb{R}} \frac{\left|e^{i\left(t^{\prime}-t^{\prime \prime}\right) \xi}-1\right|^{2}}{|\xi|^{2 H^{\prime}+1}} d \xi & =\left(\int_{\mathbb{R}} \frac{\left|e^{i \eta}-1\right|^{2}}{|\eta|^{2 H^{\prime}+1}} d \eta\right)\left|t^{\prime}-t^{\prime \prime}\right|^{2 H^{\prime}} \\
& \leq c_{1}\left|t^{\prime}-t^{\prime \prime}\right|^{2 m}
\end{aligned}
$$


where the constant $c_{1}=\int_{\mathbb{R}} \frac{\left|e^{i \eta}-1\right|^{2}}{|\eta|^{2 a+1}} d \eta+\int_{\mathbb{R}} \frac{\left|e^{i \eta}-1\right|^{2}}{|\eta|^{2 b+1}} d \eta$. Applying the Mean Value Theorem to the function $H \mapsto|\xi|^{-H-1 / 2}=e^{-(H+1 / 2) \log |\xi|}$ and using the inequalities $m \leq H^{\prime} \leq M, m \leq H^{\prime \prime} \leq M$ and $0 \leq t^{\prime \prime} \leq 1$, one obtains that

$$
\begin{aligned}
& \int_{\mathbb{R}} \frac{\left|e^{i t^{\prime \prime} \xi}-1\right|^{2}}{|\xi|}\left(\frac{1}{|\xi|^{H^{\prime}}}-\frac{1}{|\xi|^{H^{\prime \prime}}}\right)^{2} d \xi \\
& \leq 2\left(\int_{1}^{+\infty} \frac{\left|e^{i t^{\prime \prime} \xi}-1\right|^{2}}{\xi^{2 a+1}}(\log \xi)^{2} d \xi\right. \\
&\left.\quad+\int_{0}^{1} \frac{\left|e^{i t^{\prime \prime} \xi}-1\right|^{2}}{\xi^{2 b+1}}(\log \xi)^{2} d \xi\right)\left|H^{\prime}-H^{\prime \prime}\right|^{2} \\
& \leq c_{2}\left|H^{\prime}-H^{\prime \prime}\right|^{2}
\end{aligned}
$$

where the constant $c_{2}=8 \int_{1}^{+\infty} \frac{\log ^{2} \xi}{\xi^{2 a+1}} d \xi+2 \int_{0}^{1} \frac{\log ^{2} \xi}{\xi^{2 b-1}} d \xi$. It follows from Lemma 2.3 that there is $\Omega_{4}^{*}(m, M)$ an event of probability 1 (that depends on $m$ and $M)$ such that for any $\omega \in \Omega_{4}^{*}(m, M)$, the uniform Hölder exponent of the function $(t, H) \mapsto \ddot{B}_{H}(t, \omega)$ over the rectangle $[0,1] \times$ $[m, M]$ is greater than $m$. Finally, we set $\Omega_{1}^{*}=\Omega_{3}^{*} \cap\left(\cap_{(m, M) \in I} \Omega_{4}^{*}(m, M)\right)$ with $I=\{(m, M) \in \mathbb{Q} \times \mathbb{Q} ; 0<m<M<1\}$.

\section{On the global and the local regularity of the MPRE}

We study in this section how the random parameter $\{S(t)\}_{t \in[0,1]}$ affects the regularity of the MPRE $\{Z(t)\}_{t \in[0,1]}$. First we will deal with the continuity of $\{Z(t)\}_{t \in[0,1]}$, then we will determine its pointwise and uniform Hölder exponent. Let $\chi_{A}$ denote the indicator function of the set $A$.

Proposition 3.1. (a) If the trajectories of $\{S(t)\}_{t \in[0,1]}$ are continuous then those of the MPRE $\{Z(t)\}_{t \in[0,1]}$ are continuous as well.

(b) Suppose that the process $\{S(t)\}_{t \in[0,1]}$ is for all $t$ and $\omega$ of the form

$$
S(t, \omega)=\sum_{k=1}^{n} g_{k}(t) \chi_{A_{k}}(\omega),
$$

where the functions $g_{k}$ are determinitic and the events $A_{k}$ form a partition of the probability space. Then the trajectories of the $M P R E\{Z(t)\}_{t \in[0,1]}$ are continuous only when those of $\{S(t)\}_{t \in[0,1]}$ are continuous. More precisely, if a function $g_{k_{0}}$ is discontinuous at some point $t_{0}$, then the trajectories of $\{Z(t)\}_{t \in[0,1]}$ are discontinuous at $t_{0}$ with probability $P\left(A_{k_{0}}\right)$. 
Proof: Since a trajectory $t \mapsto Z(t, \omega)$ of the MPRE has been defined as the composition of the functions $f_{1}: t \mapsto(t, S(t, \omega)), f_{2}:(t, H) \mapsto$ $B_{H}(t, \omega)$, to prove (a) it is sufficient to show that these functions are continuous. The continuity of $f_{1}$ follows from that of $t \mapsto S(t, \omega)$ and the continuity of $f_{2}$ follows from Theorem 2.1.

Let us now prove (b). The function $g_{k_{0}}$ being bounded and discontinuous at $t_{0}$, there is a sequence $\left(x_{n}\right)$ converging to $t_{0}$ and such that $\lim _{n \rightarrow \infty} g_{k_{0}}\left(x_{n}\right) \neq g_{k_{0}}\left(t_{0}\right)$. Set $y_{0}=g_{k_{0}}\left(t_{0}\right)$ and $y_{1}=\lim _{n \rightarrow \infty} g_{k_{0}}\left(x_{n}\right)$. As for any $\omega \in A_{k_{0}}$ and $t \in[0,1], Z(t, \omega)=B_{g_{k_{0}}(t)}(t)$, it follows from the continuity of the function $(t, H) \mapsto B_{H}(t, \omega)$ at $\left(t_{0}, y_{1}\right)$ that for almost all $w \in A_{k_{0}}$,

$$
\lim _{n \rightarrow \infty} Z\left(x_{n}, \omega\right)=\lim _{n \rightarrow \infty} B_{g_{k_{0}}\left(x_{n}\right)}\left(x_{n}, \omega\right)=B_{y_{1}}\left(t_{0}, \omega\right) .
$$

Since $B_{y_{0}}\left(t_{0}\right)-B_{y_{1}}\left(t_{0}\right)$ is a non-degenerate Gaussian random variable, it can vanish only on a negligible event and therefore one has for almost all $\omega \in A_{k_{0}}$,

$$
Z\left(t_{0}, \omega\right)=B_{y_{0}}\left(t_{0}, \omega\right) \neq B_{y_{1}}\left(t_{0}, \omega\right) .
$$

Relations (3.2) and (3.3) imply that with probability $P\left(A_{k_{0}}\right)$ the function $t \mapsto Z(t, \omega)$ is discontinuous at $t_{0}$.

As in the case of MBM (see Relation (1.12)), in order to be able to determine the Hölder regularity of the MPRE one needs to impose a technical condition on $\beta_{S}([0,1])$, the uniform Hölder exponent over $[0,1]$ of its parameter $\{S(t)\}_{t \in[0,1]}$. We suppose that with probability 1:

$$
\sup _{t \in[0,1]} S(t, \omega)<\beta_{S}([0,1], \omega) .
$$

The following theorems describe the Hölder regularity of the MPRE.

Theorem 3.1. Let $\{Z(t)\}_{t \in[0,1]}$ be an MPRE whose parameter $\{S(t)\}_{t \in[0,1]}$ satisfies the condition $(\mathcal{C})$. Then $\left\{\alpha_{Z}(t)\right\}_{t \in(0,1)}$, the pointwise Hölder exponent of $\{Z(t)\}_{t \in(0,1)}$ (see Relation (1.7) for the definition of this exponent), can be characterized via $\{S(t)\}_{t \in(0,1)}$. Namely for any $t_{0} \in(0,1)$ one has almost surely

$$
\alpha_{Z}\left(t_{0}, \omega\right)=S\left(t_{0}, \omega\right)
$$


Theorem 3.2. Let $\{Z(t)\}_{t \in[0,1]}$ be an MPRE whose parameter $\{S(t)\}_{t \in[0,1]}$ satisfies the condition $(\mathcal{C})$. Then $\beta_{Z}(J)$, the uniform Hölder exponent of $\{Z(t)\}_{t \in[0,1]}$ over an arbitrary non-degenerate interval $J \subset[0,1]$ (see Relation (1.8) for the definition of this exponent), can be characterized via $\{S(t)\}_{t \in[0,1]}$. Namely, one has almost surely

$$
\beta_{Z}(J, \omega)=\inf _{t \in J} S(t, \omega) .
$$

To prove Theorems 3.1 and 3.2 we need the following lemma.

Lemma 3.1. Let $\{Z(t)\}_{t \in[0,1]}$ be an MPRE whose parameter $\{S(t)\}_{t \in[0,1]}$ satisfies the condition $(\mathcal{C})$. Then $\beta_{Z}(J)$, the uniform Hölder exponent of $\{Z(t)\}_{t \in[0,1]}$ over an arbitrary non-degenerate interval $J \subset[0,1]$, satisfies almost surely

$$
\inf _{t \in J} S(t, \omega) \leq \beta_{Z}(J, \omega) .
$$

To prove Lemma 3.1 we need some preliminary results.

Definition 3.1. The processes $\{\dot{Z}(t)\}_{t \in[0,1]}$ and $\{\ddot{Z}(t)\}_{t \in[0,1]}$ will be respectively the low and high frequency components of the MPRE of parameter $\{S(t)\}_{t \in[0,1]}$. They are defined as $\{Z(t)\}_{t \in[0,1]}$ but by replacing the field $\left\{B_{H}(t)\right\}_{(t, H) \in[0,1] \times[a, b]}$ respectively by its low and high frequency components, namely the fields $\left\{\dot{B}_{H}(t)\right\}_{(t, H) \in[0,1] \times[a, b]}$ and $\left\{\ddot{B}_{H}(t)\right\}_{(t, H) \in[0,1] \times[a, b]}$ that have been introduced in (2.8) and (2.9).

Remark 3.1. $\{\dot{Z}(t)\}_{t \in[0,1]}$ is more regular than $\{S(t)\}_{t \in[0,1]}$. Actually the uniform Hölder exponents of these processes over $[0,1]$ satisfy almost surely

$$
\beta_{S}([0,1]) \leq \beta_{\dot{Z}}([0,1]) .
$$

Proof of Remark 3.1: It follows from Proposition 2.1 that $(t, H) \mapsto$ $\dot{B}_{H}(t, \omega)$ is with probability 1 a Lipschitz function over $[0,1] \times[a, b]$. Therefore one has for every arbitrarily small $\epsilon>0, t^{\prime} \in[0,1]$ and $t^{\prime \prime} \in[0,1]$

$$
\begin{aligned}
\left|\dot{Z}\left(t^{\prime}, \omega\right)-\dot{Z}\left(t^{\prime \prime}, \omega\right)\right| & =\left|\dot{B}_{S\left(t^{\prime}, \omega\right)}(t, \omega)-\dot{B}_{S\left(t^{\prime \prime}, \omega\right)}\left(t^{\prime \prime}, \omega\right)\right| \\
& \leq C(\omega)\left(\left|t^{\prime}-t^{\prime \prime}\right|+\left|S\left(t^{\prime}, \omega\right)-S\left(t^{\prime \prime}, \omega\right)\right|\right) \\
& \leq C^{\prime}(\omega)\left|t^{\prime}-t^{\prime \prime}\right|^{\beta_{S}([0,1], \omega)-\epsilon},
\end{aligned}
$$

where the random variable $C$ only depends on $\epsilon$. This implies the inequality (3.7). 
Remark 3.2. To prove Lemma 3.1 and Theorems 3.1 and 3.2 one can replace the process $\{Z(t)\}_{t \in[0,1]}$ by its high frequency component $\{\ddot{Z}(t)\}_{t \in[0,1]}$.

Proof of Remark 3.2: Remark 3.2 follows from Remark 3.1 because for every $t \in[0,1]$ one has $Z(t)=\dot{Z}(t)+\ddot{Z}(t)$.

We can now prove Lemma 3.1.

Proof of Lemma 3.1: It follows from Remark 3.2 that we may replace $\{Z(t)\}_{t \in[0,1]}$ by $\{\ddot{Z}(t)\}_{t \in[0,1]}$. Suppose that $\omega \in \Omega_{1}^{*}$, the event of probability 1 , that has been introduced in Proposition 2.2 and let $m(\omega)=$ $\inf _{t \in J} S(t, \omega)$ and $M(\omega)=\sup _{t \in J} S(t, \omega)$. Setting $H^{\prime}=H^{\prime \prime}=S\left(t^{\prime}, \omega\right)$ in (2.11), we obtain that

$$
\left|\ddot{B}_{S\left(t^{\prime}, \omega\right)}\left(t^{\prime}, \omega\right)-\ddot{B}_{S\left(t^{\prime}, \omega\right)}\left(t^{\prime \prime}, \omega\right)\right| \leq C_{1}(\omega)\left|t^{\prime}-t^{\prime \prime}\right|^{m(\omega)-\epsilon},
$$

where the real $\epsilon>0$ is arbitrarily small and $C_{1}$ is a random variable that depends on $\epsilon$. Now setting $H^{\prime}=S\left(t^{\prime}, \omega\right)$ and $H^{\prime \prime}=S\left(t^{\prime \prime}, \omega\right)$ in (2.12), we obtain

$$
\begin{aligned}
\left|\ddot{B}_{S\left(t^{\prime}, \omega\right)}\left(t^{\prime \prime}, \omega\right)-\ddot{B}_{S\left(t^{\prime \prime}, \omega\right)}\left(t^{\prime \prime}, \omega\right)\right| & \leq C_{2}(\omega)\left|S\left(t^{\prime}, \omega\right)-S\left(t^{\prime \prime}, \omega\right)\right| \\
& \leq C_{2}^{\prime}(\omega)\left|t^{\prime}-t^{\prime \prime}\right|^{\beta_{S}([0,1], \omega)-\epsilon},
\end{aligned}
$$

where $\beta_{S}([0,1], \omega)$ denotes the uniform Hölder exponent of the function $t \mapsto S(t, \omega)$ over $[0,1]$. Since we assumed that $\{S(t)\}_{t \in[0,1]}$ satisfies the condition $(\mathcal{C})$, one has

$$
m(\omega) \leq \beta_{S}([0,1], \omega),
$$

and therefore (3.9) continues to hold if one replaces $\beta_{S}$ by $m(\omega)$. The inequalities (3.8) and (3.9) then imply

$$
\begin{aligned}
\left|\ddot{Z}\left(t^{\prime}, \omega\right)-\ddot{Z}\left(t^{\prime \prime}, \omega\right)\right|= & \left|\ddot{B}_{S\left(t^{\prime}, \omega\right)}\left(t^{\prime}, \omega\right)-\ddot{B}_{S\left(t^{\prime \prime}, \omega\right)}\left(t^{\prime \prime}, \omega\right)\right| \\
\leq & \left|\ddot{B}_{S\left(t^{\prime}, \omega\right)}\left(t^{\prime}, \omega\right)-\ddot{B}_{S\left(t^{\prime}, \omega\right)}\left(t^{\prime \prime}, \omega\right)\right| \\
& +\left|\ddot{B}_{S\left(t^{\prime}, \omega\right)}\left(t^{\prime \prime}, \omega\right)-\ddot{B}_{S\left(t^{\prime \prime}, \omega\right)}\left(t^{\prime \prime}, \omega\right)\right| \\
\leq & C_{2}^{\prime \prime}(\omega)\left|t^{\prime}-t^{\prime \prime}\right|^{m(\omega)-\epsilon} .
\end{aligned}
$$

To prove Theorem 3.1 we need some additional results. 
Lemma 3.2. Fix $t_{0} \in[0,1]$ and $\omega \in \Omega_{3}^{*}$, the event of probability 1 introduced in Lemma 2.2. Then the series

$$
\ddot{T}_{t_{0}}(t, \omega)=\sum_{j=0}^{\infty} \sum_{k \in \mathbb{Z}} 2^{-j S\left(t_{0}, \omega\right)} \epsilon_{j, k}(\omega) \Psi\left(2^{j} t-k, S\left(t_{0}, \omega\right)\right),
$$

is uniformly convergent in $t$ on every compact of $\mathbb{R}$. In addition, there is a random variable $C_{5}>0$ such that for every $t \in \mathbb{R}$ and $\omega \in \Omega_{3}^{*}$, one has

$$
\left|\ddot{T}_{t_{0}}(t, \omega)\right| \leq C_{5}(\omega) \sqrt{\log (2+|t|)} .
$$

Proof of Lemma 3.2: To show that the series (3.11) is uniformly convergent in $t$ on every compact of $\mathbb{R}$ one uses the inequalities (2.7) and (2.13) and the same techniques as in Section 3 in [3]. Let us now prove that the inequality (3.12) holds. Using again the inequalities (2.7) and (2.13) one obtains that for every $t \in \mathbb{R}$

$$
\left|\ddot{T}_{t_{0}}(t, \omega)\right| \leq C(\omega) \sum_{j=0}^{\infty} 2^{-j S\left(t_{0}, \omega\right)} \log ^{1 / 2}(2+j) \sum_{k \in \mathbb{Z}} \frac{\log ^{1 / 2}(2+|k|)}{\left(2+\left|2^{j} t-k\right|\right)^{2}}
$$

Let $\left[2^{j} t\right]$ denote the integer part of $2^{j} t$. Using the sub-additivity of the function $x \mapsto \log ^{1 / 2}(2+x)$ (see Lemma 3.5 of [3] ) and the inequality $a+b \leq a b$ which holds for all reals $a \geq 2$ and $b \geq 2$, we have that

$$
\begin{aligned}
\sum_{k \in \mathbb{Z}} \frac{\log ^{1 / 2}(2+|k|)}{\left(2+\left|2^{j} t-k\right|\right)^{2}} \leq & \sum_{k \in \mathbb{Z}} \frac{\log ^{1 / 2}\left(9+\left|k+\left[2^{j} t\right]\right|\right)}{\left(2+\left|2^{j} t-\left[2^{j} t\right]-k\right|\right)^{2}} \\
\leq & \sum_{k \in \mathbb{Z}} \frac{\log ^{1 / 2}(9+|k|)}{\left(2+\left|2^{j} t-\left[2^{j} t\right]-k\right|\right)^{2}} \\
& +\sum_{k \in \mathbb{Z}} \frac{\log ^{1 / 2}\left(9+2^{j}\right)}{\left(2+\left|2^{j} t-\left[2^{j} t\right]-k\right|\right)^{2}} \\
& +\sum_{k \in \mathbb{Z}} \frac{\log ^{1 / 2}(9+|t|)}{\left(2+\left|2^{j} t-\left[2^{j} t\right]-k\right|\right)^{2}} \\
\leq & c^{\prime} \log ^{1 / 2}\left(9+2^{j}\right) \log ^{1 / 2}(9+|t|),
\end{aligned}
$$

where the constant $c^{\prime}=2 \sup _{x \in[0,1]}\left(\sum_{k \in \mathbb{Z}} \frac{\log ^{1 / 2}(9+|k|)}{(2+|x-k|)^{2}}\right)$. Thus (3.12) follows from (3.13) and (3.14). 
Lemma 3.3. Fix $t_{0} \in(0,1)$ and let $\left\{\alpha_{\ddot{T}_{t_{0}}}(t)\right\}_{t \in \mathbb{R}}$ and $\left\{\alpha_{\ddot{Z}}(t)\right\}_{t \in(0,1)}$ be respectively the pointwise Hölder exponents of the processes $\left\{\ddot{T}_{t_{0}}(t)\right\}_{t \in \mathbb{R}}$ and $\{\ddot{Z}(t)\}_{t \in(0,1)}$. Then for any $\omega \in \Omega_{1}^{*}$, the event with probability 1 that has been introduced in Proposition 2.2, one has

$$
\alpha_{\ddot{Z}}\left(t_{0}, \omega\right) \leq S\left(t_{0}, \omega\right) \quad \text { if and only if } \quad \alpha_{\ddot{T}_{t_{0}}}\left(t_{0}, \omega\right) \leq S\left(t_{0}, \omega\right) .
$$

Proof of Lemma 3.3: Since $\beta_{S}([0,1])$, the uniform Hölder exponent over $[0,1]$ of the process $\{S(t)\}_{t \in[0,1]}$, satisfies the condition $(\mathcal{C})$, it is sufficient to prove that for any $\omega \in \Omega_{1}^{*}$ and any $\alpha$ in the interval $\left(0, \beta_{S}([0,1], \omega)\right)$ one has

$$
\limsup _{h \rightarrow 0} \frac{\left|\ddot{T}_{t_{0}}\left(t_{0}+h, \omega\right)-\ddot{T}_{t_{0}}\left(t_{0}, \omega\right)\right|}{|h|^{\alpha}}=0
$$

if and only if

$$
\limsup _{h \rightarrow 0} \frac{\left|\ddot{Z}\left(t_{0}+h, \omega\right)-\ddot{Z}\left(t_{0}, \omega\right)\right|}{|h|^{\alpha}}=0 .
$$

It follows from Definition 3.1 that for any $t_{0}+h \in[0,1]$, one has

$$
\begin{aligned}
\mid \ddot{B}_{S\left(t_{0}, \omega\right)}\left(t_{0}+h, \omega\right) & -\ddot{B}_{S\left(t_{0}, \omega\right)}\left(t_{0}, \omega\right) \mid \\
& -\left|\ddot{B}_{S\left(t_{0}+h, \omega\right)}\left(t_{0}+h, \omega\right)-\ddot{B}_{S\left(t_{0}, \omega\right)}\left(t_{0}+h, \omega\right)\right| \\
\leq & \left|\ddot{Z}\left(t_{0}+h, \omega\right)-\ddot{Z}\left(t_{0}, \omega\right)\right| \\
\leq & \left|\ddot{B}_{S\left(t_{0}, \omega\right)}\left(t_{0}+h, \omega\right)-\ddot{B}_{S\left(t_{0}, \omega\right)}\left(t_{0}, \omega\right)\right| \\
& +\left|\ddot{B}_{S\left(t_{0}+h, \omega\right)}\left(t_{0}+h, \omega\right)-\ddot{B}_{S\left(t_{0}, \omega\right)}\left(t_{0}+h, \omega\right)\right| .
\end{aligned}
$$

On the one hand, using the Relation (2.12) and the definition of the uniform Hölder exponent we obtain that

$$
\begin{aligned}
\left|\ddot{B}_{S\left(t_{0}+h, \omega\right)}\left(t_{0}+h, \omega\right)-\ddot{B}_{S\left(t_{0}, \omega\right)}\left(t_{0}+h, \omega\right)\right| \\
\leq \sup _{t \in[0,1]}\left|\ddot{B}_{S\left(t_{0}+h, \omega\right)}(t, \omega)-\ddot{B}_{S\left(t_{0}, \omega\right)}(t, \omega)\right| \\
\leq C_{2}(\omega)\left|S\left(t_{0}+h, \omega\right)-S\left(t_{0}, \omega\right)\right| \\
\leq C(\omega)|h|^{\beta_{S}([0,1], \omega)-\epsilon},
\end{aligned}
$$

where the real $\epsilon>0$ is arbitrarily small. On the other hand, Relations (2.9) and (3.11) imply that

(3.20) $\left|\ddot{T}_{t_{0}}\left(t_{0}+h, \omega\right)-\ddot{T}_{t_{0}}\left(t_{0}, \omega\right)\right|=\left|\ddot{B}_{S\left(t_{0}, \omega\right)}\left(t_{0}+h, \omega\right)-\ddot{B}_{S\left(t_{0}, \omega\right)}\left(t_{0}, \omega\right)\right|$. 
Thus, it follows from (3.18), (3.19) and (3.20) that

$$
\begin{aligned}
\mid \ddot{T}_{t_{0}}\left(t_{0}+h, \omega\right) & -\ddot{T}_{t_{0}}\left(t_{0}, \omega\right) \mid \\
& -C(\omega)|h|^{\beta_{S}([0,1], \omega)-\epsilon} \\
\leq & \left|\ddot{Z}\left(t_{0}+h, \omega\right)-\ddot{Z}\left(t_{0}, \omega\right)\right| \\
\leq & \left|\ddot{T}_{t_{0}}\left(t_{0}+h, \omega\right)-\ddot{T}_{t_{0}}\left(t_{0}, \omega\right)\right| \\
& +C(\omega)|h|^{\beta_{S}([0,1], \omega)-\epsilon},
\end{aligned}
$$

which proves that the Relations (3.16) and (3.17) are equivalent.

Lemma 3.4. Let $\widetilde{\Psi}$ be a function defined for every $(x, H) \in \mathbb{R} \times[a, b]$ as

$$
\widetilde{\Psi}(x, H)=i \int_{\mathbb{R}} e^{i x \xi} \xi|\xi|^{H-1 / 2} \widehat{\psi}(\xi) d \xi .
$$

This function has the following properties:

(i) $\widetilde{\Psi}$ is localized in the variable $x$ uniformly in the variable $H$. More precisely, there is a constant $c>0$ (that only depends on a and $b$ ) such that for any $(x, H) \in \mathbb{R} \times[a, b]$ one has

$$
|\widetilde{\Psi}(x, H)| \leq c(2+|x|)^{-2} .
$$

(ii) For any $H \in[a, b]$, the first moment of the function $\widetilde{\Psi}(., H)$ vanishes, that is,

$$
\int_{\mathbb{R}} \widetilde{\Psi}(x, H) d x=0 .
$$

(iii) Let $\Psi$ be the function introduced in (2.5). For any $H \in[a, b]$, the system of functions $\left\{2^{j / 2} \Psi\left(2^{j} t-k, H\right) ; j \in \mathbb{N}\right.$ and $\left.k \in \mathbb{Z}\right\}$ and $\left\{2^{j / 2} \widetilde{\Psi}\left(2^{j} t-k, H\right) ; j \in \mathbb{N}\right.$ and $\left.k \in \mathbb{Z}\right\}$ is biorthogonal. This means that for any $j \in \mathbb{N}, j^{\prime} \in \mathbb{N}, k \in \mathbb{Z}$ and $k^{\prime} \in \mathbb{Z}$, one has

$$
2^{\left(j+j^{\prime}\right) / 2} \int_{\mathbb{R}} \Psi\left(2^{j} t-k, H\right) \overline{\widetilde{\Psi}\left(2^{j^{\prime}} t-k^{\prime}, H\right)} d t=\delta\left(j, k ; j^{\prime}, k^{\prime}\right),
$$

where $\delta\left(j, k ; j^{\prime}, k^{\prime}\right)=1$ if $(j, k)=\left(j^{\prime}, k^{\prime}\right)$ and 0 otherwise.

Proof of Lemma 3.4: Part (i) can be shown as in Lemma 2.1. To prove Parts (ii) and (iii) let us first observe that for every $H \in[a, b]$ the Fourier transforms of the functions $x \mapsto \Psi(x, H)$ and $x \mapsto \widetilde{\Psi}(x, H)$ are respectively the functions $\xi \mapsto \frac{\widehat{\psi}(\xi)}{i \xi|\xi|^{H-1 / 2}}$ and $\xi \mapsto i \xi|\xi|^{H-1 / 2} \widehat{\psi}(\xi)$. As $\xi \mapsto i \xi|\xi|^{H-1 / 2} \widehat{\psi}(\xi)$ vanishes in a neighbourhood of the origin one 
gets (ii). Part (iii) can be obtained as follows. Using Parseval Formula we have that

$$
\begin{aligned}
& 2^{\left(j+j^{\prime}\right) / 2} \int_{\mathbb{R}} \Psi\left(2^{j} t-k, H\right) \widetilde{\widetilde{\Psi}\left(2^{j^{\prime}} t-k^{\prime}, H\right)} d t \\
= & 2^{-\left(j+j^{\prime}\right) / 2} \int_{\mathbb{R}} e^{-i\left(k / 2^{j}-k^{\prime} / 2^{j^{\prime}}\right) \xi} \frac{\left|2^{-j^{\prime}} \xi\right|^{H+1 / 2}}{\left|2^{-j} \xi\right|^{H+1 / 2}} \widehat{\psi}\left(2^{-j} \xi\right) \overline{\widehat{\psi}\left(2^{-j^{\prime}} \xi\right)} d \xi \\
= & 2^{\left(j-j^{\prime}\right)(H+1 / 2)} \times 2^{-\left(j+j^{\prime}\right) / 2} \int_{\mathbb{R}} e^{-i\left(k / 2^{j}-k^{\prime} / 2^{j^{\prime}}\right) \xi} \widehat{\psi}\left(2^{-j} \xi\right) \overline{\widehat{\psi}\left(2^{-j^{\prime}} \xi\right)} d \xi \\
= & 2^{\left(j-j^{\prime}\right)(H+1 / 2)} \times 2^{\left(j+j^{\prime}\right) / 2} \int_{\mathbb{R}} \psi\left(2^{j} t-k\right) \overline{\psi\left(2^{j^{\prime}} t-k^{\prime}\right)} d t \\
= & \delta\left(j, k ; j^{\prime}, k^{\prime}\right) .
\end{aligned}
$$

Observe that the last equality follows from the orthonormality of the functions $2^{j / 2} \psi\left(2^{j} t-k\right)$ (see the beginning of Section 2).

Now we are able to prove Theorems 3.1 and 3.2.

Proof of Theorem 3.1: Since the function $t \mapsto S(t, \omega)$ is continuous over the interval $[0,1]$ with probability 1 , there is, for any $t_{0} \in(0,1)$ and any arbitrarily small $\epsilon>0$, an $\eta>0$, such that

$$
\inf _{t \in\left[t_{0}-\eta, t_{0}+\eta\right]} S(t, \omega) \geq S\left(t_{0}, \omega\right)-\epsilon .
$$

Lemma 3.1 and inequality (1.9) thus imply that almost surely

$$
\alpha_{Z}\left(t_{0}, \omega\right) \geq S\left(t_{0}, \omega\right)-\epsilon .
$$

Letting $\epsilon \rightarrow 0$ we obtain that, with probability 1 ,

$$
\alpha_{Z}\left(t_{0}, \omega\right) \geq S\left(t_{0}, \omega\right) .
$$

Let us now show that, almost surely, $\alpha_{Z}\left(t_{0}, \omega\right) \leq S\left(t_{0}, \omega\right)$. It follows from Remark 3.2 and Lemma 3.3 that one can prove instead

$$
\alpha_{\ddot{T}_{t_{0}}}\left(t_{0}, \omega\right) \leq S\left(t_{0}, \omega\right) .
$$

To establish this last inequality, suppose ad absurdum that there is a non-negligible event $A$ such that for any $\omega \in A$ one has

$$
\alpha_{\ddot{T}_{t_{0}}}\left(t_{0}, \omega\right)>S\left(t_{0}, \omega\right) .
$$

We will use a method which allowed Jaffard [7] to obtain a wavelet characterization of the pointwise Hölder exponent. Let $\Omega_{3}^{*}$ be the event 
of probability 1 that has been introduced in Lemma 2.2. Relations (3.12) and (3.22) imply that for any $j \in \mathbb{N}, k \in \mathbb{Z}$ and $\omega \in \Omega_{3}^{*} \cap A$, the integral

$$
I_{j, k}(\omega)=2^{j} \int_{\mathbb{R}} \ddot{T}_{t_{0}}(t, \omega) \overline{\widetilde{\Psi}\left(2^{j} t-k, S\left(t_{0}, \omega\right)\right)} d t,
$$

is convergent and Relations (3.11) and (3.24) imply that

$$
I_{j, k}(\omega)=2^{-j S\left(t_{0}, \omega\right)} \epsilon_{j, k}(\omega) .
$$

Choose $\eta>0$ such that

$$
S\left(t_{0}, \omega\right)+\eta<\min \left(\alpha_{\ddot{T}_{t_{0}}}\left(t_{0}, \omega\right), 1\right) .
$$

Using the definition of the pointwise Hölder exponent, namely Relation (1.7) one obtains that for any $\left|t-t_{0}\right|$ small enough

$$
\left|\ddot{T}_{t_{0}}(t, \omega)-\ddot{T}_{t_{0}}\left(t_{0}, \omega\right)\right| \leq C(\omega)\left|t-t_{0}\right|^{S\left(t_{0}, \omega\right)+\eta}
$$

and thanks to Relation (3.12) this inequality remains true for any $t \in \mathbb{R}$. Then it follows from Relations (3.22), (3.23), (3.27) and (3.30) that for any $j \in \mathbb{N}, k \in \mathbb{Z}$ and $\omega \in \Omega_{3}^{*} \cap A$, one has

$$
\begin{aligned}
\left|I_{j, k}(\omega)\right| & =2^{j}\left|\int_{\mathbb{R}}\left(\ddot{T}_{t_{0}}(t, \omega)-\ddot{T}_{t_{0}}\left(t_{0}, \omega\right)\right) \overline{\widetilde{\Psi}\left(2^{j} t-k, S\left(t_{0}, \omega\right)\right)} d t\right| \\
& \leq 2^{j} \int_{\mathbb{R}}\left|\ddot{T}_{t_{0}}(t, \omega)-\ddot{T}_{t_{0}}\left(t_{0}, \omega\right)\right|\left|\widetilde{\Psi}\left(2^{j} t-k, S\left(t_{0}, \omega\right)\right)\right| d t \\
& \leq C^{\prime}(\omega) 2^{j} \int_{\mathbb{R}} \frac{\left|t-t_{0}\right|^{S\left(t_{0}, \omega\right)+\eta}}{\left(2+\left|2^{j} t-k\right|\right)^{2}} d t .
\end{aligned}
$$

Setting $u=2^{j} t-k$ in this last integral yields

$$
\left|I_{j, k}(\omega)\right| \leq C^{\prime}(\omega) \int_{\mathbb{R}} \frac{\left|2^{-j}(u+k)-t_{0}\right|^{S\left(t_{0}, \omega\right)+\eta}}{(2+|u|)^{2}} d u .
$$

Since $\left|2^{-j}(u+k)-t_{0}\right|^{S\left(t_{0}, \omega\right)+\eta} \leq\left|2^{-j} u\right|^{S\left(t_{0}, \omega\right)+\eta}+\left|t_{0}-2^{-j} k\right|^{S\left(t_{0}, \omega\right)+\eta}$, one obtains

$$
\left|I_{j, k}(\omega)\right| \leq C^{\prime \prime}(\omega) 2^{-j\left(S\left(t_{0}, \omega\right)+\eta\right)}\left(1+\left|2^{j} t_{0}-k\right|^{S\left(t_{0}, \omega\right)+\eta}\right),
$$

where $C^{\prime \prime}(\omega)=C^{\prime}(\omega)\left(\int_{\mathbb{R}} \frac{|u|^{S\left(t_{0}, \omega\right)+\eta}}{(2+|u|)^{2}} d u+\int_{\mathbb{R}} \frac{d u}{(2+|u|)^{2}}\right)$. Relations (3.28) and (3.31) imply

$$
\left|\epsilon_{j, k}(\omega)\right| \leq C^{\prime \prime}(\omega) 2^{-j \eta}\left(1+\left|2^{j} t_{0}-k\right|^{S\left(t_{0}, \omega\right)+\eta}\right),
$$

for all $\omega \in \Omega_{3}^{*} \cap A, j \in \mathbb{N}$ and $k \in \mathbb{Z}$. Suppose now that $n \mapsto\left(j_{n}, k_{n}\right)$ is a sequence with values in $\mathbb{N} \times \mathbb{Z}$ satisfying for all $n \in \mathbb{N}, j_{n} \geq n$ and $\left|2^{j_{n}} t_{0}-k_{n}\right| \leq 1$. To simplify our notations we set $\epsilon_{n}=\epsilon_{j_{n}, k_{n}}$ for 
every $n \in \mathbb{N}$. Observe that the inequality (3.32) entails that for all $n \in \mathbb{N}$ and $\omega \in \Omega_{3}^{*} \cap A$,

$$
\left|\epsilon_{n}(\omega)\right| \leq C^{\prime \prime}(\omega) 2^{-n \eta+1} .
$$

On the other hand, since for each $n, \epsilon_{n}$ is a standard random Gaussian variable, one has $P\left(\left|\epsilon_{n}\right| \geq 1\right)>0$ and thus $\sum_{n=0}^{\infty} P\left(\left|\epsilon_{n}\right| \geq 1\right)=\infty$. Since the random variables $\epsilon_{n}$ are independent, the Borel-Cantelli lemma implies that there is $\Omega_{6}^{*}$, an event of probability 1 , with the following property: for any $\omega \in \Omega_{6}^{*}$ there is a subsequence $l \mapsto n_{l}$ such that for every $l$,

$$
\left|\epsilon_{n_{l}}(\omega)\right| \geq 1 .
$$

Taken together, Relations (3.33) and (3.34) imply that for any $l \in \mathbb{N}$ and $\omega \in \Omega_{3}^{*} \cap A \cap \Omega_{6}^{*}$ one has $1 \leq C^{\prime \prime}(\omega) 2^{-n_{\iota} \eta+1}$, which is a contradiction.

Proof of Theorem 3.2: This theorem follows from Theorem 3.1, Relation (1.9) and Lemma 3.1.

Proposition 3.2. If $S$ is a non-degenerate random process, then the resulting MPRE is a non-Gaussian stochastic process.

This follows from Theorem 3.1 and the following lemma.

Lemma 3.5. If $\{X(t)\}_{t \in[0,1]}$ is a Gaussian process with continuous and nowhere differentiable trajectories, then $\alpha_{X}(t)$, its pointwise Hölder exponent at an arbitrary $t$, is almost surely deterministic.

Proof of Lemma 3.5: Let $0<s_{1} \leq s_{2}$ be such that

$$
P\left(s_{1} \leq \alpha_{X}(t)\right)>0 \text { and } P\left(\alpha_{X}(t) \leq s_{2}\right)>0,
$$

and for $k \in\{1,2\}$, let $\left\{Y_{t, k}(u)\right\}_{u \in[0,1]}$ be the Gaussian process defined as

$$
Y_{t, k}(u)= \begin{cases}0, & \text { if } u=t \\ \frac{X(u)-X(t)}{|u-t|^{s_{k}}}, & \text { otherwise. }\end{cases}
$$

It follows from Relation (1.7) and the continuity of the process $\{X(t)\}_{t \in[0,1]}$ that one has $P\left(\sup _{u \in[0,1]}\left|Y_{t, 1}(u)\right|<\infty\right)>0$ and $P\left(\sup _{u \in[0,1]}\left|Y_{t, 2}(u)\right|=\infty\right)>0$. Then using Proposition 1, p. 211 of [11] one gets that $P\left(\sup _{u \in[0,1]}\left|Y_{t, 1}(u)\right|<\infty\right)=1$ and $P\left(\sup _{u \in[0,1]}\left|Y_{t, 2}(u)\right|=\right.$ $\infty)=1$. This means that $P\left(s_{1} \leq \alpha_{X}(t)\right)=1$ and $P\left(\alpha_{X}(t) \leq s_{2}\right)=1$. Set now

$$
h(t)=\sup \left\{s_{1}>0 ; P\left(s_{1} \leq \alpha_{X}(t)\right)=1\right\} .
$$


Then for every $s_{2}>h(t)$ one has $P\left(\alpha_{X}(t) \leq s_{2}\right)=1$ and hence

$$
h(t)=\inf \left\{s_{2} ; P\left(\alpha_{X}(t) \leq s_{2}\right)=1\right\} .
$$

Relations (3.37) and (3.38) imply that with probability $1, \alpha_{X}(t)=h(t)$.

\section{Self-similarity and stationarity of the increments of some classes of MPRE}

In this section, we give sufficient conditions for the MPRE to be selfsimilar (in the sense of marginal distributions) or have stationary increments. In the following theorem we suppose for convenience that the MPRE is defined on the whole real line and not only on the interval $[0,1]$.

Theorem 4.1. Let $\{Z(t)\}_{t \in \mathbb{R}}$ be an MPRE whose parameter $\{S(t)\}_{t \in \mathbb{R}}$ is a stationary stochastic process independent of the white noise. Then $\{Z(t)\}_{t \in \mathbb{R}}$ satisfies the following self-similarity property. For any reals $a>0$ and $t$, one has

$$
Z(a t) \stackrel{\left(d_{1}\right)}{=} a^{S(t)} Z(t)
$$

where $\stackrel{\left(d_{1}\right)}{=}$ means equality of the marginal distributions.

To prove this theorem we use Auscher's wavelet bases [1]. Namely, wavelet bases with rational dilation factor that share the same properties as Lemarié-Meyer wavelet bases. Auscher has shown in [1] that the following result holds.

Lemma 4.1 (Auscher). Consider an arbitrary rational $p / q>1$, where $p$ and $q$ being relatively prime integers. Then there are functions $\psi_{1}, \ldots, \psi_{p-q}$ in $S(\mathbb{R})$ whose Fourier transforms are compactly supported and vanish in a neighbourhood of the origin, such that $\left\{(p / q)^{j / 2} \psi_{l}\left((p / q)^{j} x-k q\right) ; j, k \in \mathbb{Z}, 1 \leq l \leq p-q\right\}$ is an orthonormal basis of $L^{2}(\mathbb{R})$.

We now introduce a wavelet decomposition with rational dilation factor for the random field $\left\{B_{H}(t)\right\}_{(t, H) \in \mathbb{R} \times[a, b]}$ and for the MPRE $\{Z(t)\}_{t \in \mathbb{R}}=\left\{B_{S(t)}(t)\right\}_{t \in \mathbb{R}}$. 
Proposition 4.1. Consider an arbitrary rational $p / q>1$, where $p$ and $q$ being relatively prime. Then the field $\left\{B_{H}(t)\right\}_{(t, H) \in \mathbb{R} \times[a, b]}$ can be expressed as the random series

$$
\begin{aligned}
B_{H}(t, \omega)=\sum_{l=1}^{p-q} & \sum_{j=-\infty}^{\infty} \sum_{k=-\infty}^{\infty}(p / q)^{-j H} \\
& \times\left(\Psi_{l}\left((p / q)^{j} t-k q, H\right)-\Psi_{l}(-k q, H)\right) \epsilon_{l, j, k}(\omega),
\end{aligned}
$$

where $\left\{\epsilon_{l, j, k}\right\}$ is a sequence of $\mathcal{N}(0,1)$ Gaussian random variables and where for every $1 \leq l \leq p-q$ and $(x, H) \in \mathbb{R} \times[a, b]$

$$
\Psi_{l}(x, H)=\int_{\mathbb{R}} e^{i x \xi} \frac{\widehat{\psi}_{l}(\xi)}{i \xi|\xi|^{H-1 / 2}} d \xi,
$$

$\psi_{1}, \ldots, \psi_{p-q}$ being Auscher mother wavelets that generate a basis of dilation factor $p / q$. Then $\Psi_{1}, \ldots, \Psi_{p-q}$ are $C^{\infty}$ functions over $\mathbb{R} \times[a, b]$ and their partial derivatives of any order are localized in $x$ uniformly in $H$. Thus the series (4.2) is with probability 1 , uniformly convergent in $(t, H)$ on every compact of $\mathbb{R} \times[a, b]$.

Proof of Proposition 4.1: This proposition can be proved by using the same techniques as in Section 2.

Remark 4.1. The MPRE $\{Z(t)\}_{t \in \mathbb{R}}$ with parameter $\{S(t)\}_{t \in \mathbb{R}}$ can be expressed as the random series

$$
\begin{aligned}
Z(t, \omega) & =\sum_{l=1}^{p-q} \sum_{j=-\infty}^{\infty} \sum_{k=-\infty}^{\infty}(p / q)^{-j S(t, \omega)} \\
& \times\left(\Psi_{l}\left((p / q)^{j} t-k q, S(t, \omega)\right)-\Psi_{l}(-k q, S(t, \omega))\right) \epsilon_{l, j, k}(\omega),
\end{aligned}
$$

which is, with probability 1 , uniformly convergent in $t$ on every compact of $\mathbb{R}$.

Proof of Remark 4.1: This remark is a straightforward consequence of Definition 1.1 and Proposition 4.1.

Now we are able to prove Theorem 4.1.

Proof of Theorem 4.1: First we will suppose that $a$ is a rational number greater than 1 . We therefore have $a=p / q, p>q>0$ being relatively 
prime integers. As the stochastic process $\{S(t)\}_{t \in \mathbb{R}}$ is stationary and independent of the white noise it follows that for every $x \in \mathbb{R}$,

$$
(S(x), Z(x)) \stackrel{(a . s)}{=}\left(S(x), B_{S(x)}(x)\right) \stackrel{\left(d_{1}\right)}{=}\left(S(0), B_{S(0)}(x)\right) .
$$

Then Relations (4.2), (4.4) and (4.5) imply that for every $t \in \mathbb{R}$,

$$
\begin{aligned}
Z\left(\frac{p}{q} t\right) \stackrel{\stackrel{\left(d_{1}\right)}{=}}{ } B_{S(0)}\left(\frac{p}{q} t\right) \\
\stackrel{(a . s)}{=} \sum_{l=1}^{p-q} \sum_{j, k \in \mathbb{Z}}(p / q)^{-j S(0)} \\
\quad \times\left(\Psi_{l}\left((p / q)^{j+1} t-k q, S(0)\right)-\Psi_{l}(-k q, S(0))\right) \epsilon_{l, j, k} \\
\stackrel{(a . s)}{=}(p / q)^{S(0)} B_{S(0)}(t) \stackrel{\left(d_{1}\right)}{=}(p / q)^{S(t)} B_{S(t)}(t) \stackrel{(a . s)}{=}(p / q)^{S(t)} Z(t) .
\end{aligned}
$$

Now suppose that $a$ is a positive rational number lower than 1 . We therefore have $a=q / p, p>q>0$ being relatively prime integers. It follows from Relations (4.2), (4.4) and (4.5) that for every $t \in \mathbb{R}$,

$$
\begin{aligned}
Z\left(\frac{q}{p} t\right) \stackrel{\stackrel{\left(d_{1}\right)}{=}}{ } B_{S(0)}\left(\frac{q}{p} t\right) \\
\stackrel{(a . s)}{=} \sum_{l=1}^{p-q} \sum_{j, k \in \mathbb{Z}}(p / q)^{-j S(0)} \\
\quad \times\left(\Psi_{l}\left((p / q)^{j-1} t-k q, S(0)\right)-\Psi_{l}(-k q, S(0))\right) \epsilon_{l, j, k} \\
\stackrel{(a . s)}{=}(q / p)^{S(0)} B_{S(0)}(t) \stackrel{\left(d_{1}\right)}{=}(q / p)^{S(t)} B_{S(t)}(t) \stackrel{(a . s)}{=}(q / p)^{S(t)} Z(t) .
\end{aligned}
$$

Finally suppose that $a$ is a positive irrational number. Let $\left(a_{n}\right)$ be a sequence of positive rationals converging to $a$. One has almost surely for every real $t$,

$$
a^{S(t)} Z(t)=\lim _{n \rightarrow \infty} a_{n}^{S(t)} Z(t)
$$

and

$$
Z(a t)=\lim _{n \rightarrow \infty} Z\left(a_{n} t\right)
$$


because of the continuity of the process $\{Z(t)\}_{t \in[0,1]}$. Since our previous results imply that for every $n$,

$$
Z\left(a_{n} t\right) \stackrel{\left(d_{1}\right)}{=} a_{n}^{S(t)} Z(t)
$$

it follows from (4.6), (4.7) and (4.8) that $Z(a t) \stackrel{\left(d_{1}\right)}{=} a^{S(t)} Z(t)$.

We now give a sufficient condition for the MPRE to have stationary increments.

Theorem 4.2. Let $\{Z(t)\}_{t \in[0,1]}$ be an MPRE whose parameter $S$ is a random variable independent of the white noise. Then the increments of $\{Z(t)\}_{t \in[0,1]}$ are stationary. Namely, for any $t \in(0,1)$, one has

$$
\{Z(t+h)-Z(t)\}_{h \in[0,1-t]} \stackrel{(d)}{=}\{Z(h)-Z(0)\}_{h \in[0,1-t]},
$$

where $\stackrel{(d)}{=}$ means equality of the finite-dimensional distributions.

Proof of Theorem 4.2: Suppose first that the random variable $S$ takes a finite number of values $\alpha_{1}, \ldots, \alpha_{n}$. Since for any $i=1, \ldots, n$, the process $\left\{B_{\alpha_{i}}(t)\right\}_{t \in[0,1]}$ has stationary increments and is independent of $S$, it follows from Definition 1.1 that for any integer $K \geq 1$, reals $\theta_{1}, \ldots, \theta_{K}$, $t \in[0,1], t+h_{k} \in[0,1], k=1, \ldots, K$, and for any Borel set $D$,

$$
\begin{aligned}
& P\left[\sum_{k=1}^{K}\left(\theta_{k}\left(Z\left(t+h_{k}\right)-Z(t)\right) \in D\right) \cap\left(S=\alpha_{i}\right)\right] \\
= & P\left[\sum_{k=1}^{K}\left(\theta_{k}\left(B_{\alpha_{i}}\left(t+h_{k}\right)-B_{\alpha_{i}}(t)\right) \in D\right) \cap\left(S=\alpha_{i}\right)\right] \\
= & P\left[\sum_{k=1}^{K} \theta_{k}\left(B_{\alpha_{i}}\left(t+h_{k}\right)-B_{\alpha_{i}}(t)\right) \in D\right] P\left(S=\alpha_{i}\right) \\
= & P\left[\sum_{k=1}^{K} \theta_{k}\left(B_{\alpha_{i}}\left(h_{k}\right)-B_{\alpha_{i}}(0)\right) \in D\right] P\left(S=\alpha_{i}\right) \\
= & P\left[\sum_{k=1}^{K}\left(\theta_{k}\left(B_{\alpha_{i}}\left(h_{k}\right)-B_{\alpha_{i}}(0)\right) \in D\right) \cap\left(S=\alpha_{i}\right)\right] \\
= & P\left[\sum_{k=1}^{K}\left(\theta_{k}\left(Z\left(h_{k}\right)-Z(0)\right) \in D\right) \cap\left(S=\alpha_{i}\right)\right] .
\end{aligned}
$$


Since $\left\{\left(S=\alpha_{i}\right)\right\}_{1 \leq i \leq n}$ partitions the probability space, we get

$$
\begin{aligned}
& P\left[\sum_{k=1}^{K} \theta_{k}\left(Z\left(t+h_{k}\right)-Z(t)\right) \in D\right] \\
= & P\left[\sum_{k=1}^{K} \theta_{k}\left(Z\left(h_{k}\right)-Z(0)\right) \in D\right] .
\end{aligned}
$$

We want to show that Relation (4.9) holds for general $S$. Approximate $S$ by a sequence of random variables $\left(S_{n}\right)_{n \geq 1}$ that take a finite number of values and are independent of the white noise. For every $n \geq 1$, define $S_{n}$ as

$$
S_{n}=\sum_{k=1}^{n}\left(a+k \frac{(b-a)}{n}\right) \chi_{\left[a+(k-1) \frac{(b-a)}{n}, a+k \frac{(b-a)}{n}\right)}(S)+\chi_{\{b\}}(S),
$$

where $\chi_{A}$ denotes the indicator function of the set $A$. It is clear that the sequence $\left(S_{n}\right)$ converges to $S$ almost surely. Let $\left\{Z_{n}(t)\right\}_{t \in[0,1]}$ denote the sequence of MPREs whose parameters are the random variables $S_{n}$. It follows from Theorem 2.1 (and Relation (2.12)) that almost surely for any $t \in[0,1]$,

$\left|Z(t)-Z_{n}(t)\right|=\left|B_{S}(t)-B_{S_{n}}(t)\right| \leq \sup _{x \in[0,1]}\left|B_{S}(x)-B_{S_{n}}(x)\right| \leq C\left|S-S_{n}\right|$,

and hence

$$
\lim _{n \rightarrow \infty} Z_{n}(t, \omega)=Z(t, \omega) .
$$

Finally, since for every integer $n, t \in(0,1)$, one has $\left\{Z_{n}(t+h)-\right.$ $\left.Z_{n}(t)\right\}_{h \in[0,1-t]} \stackrel{(d)}{=}\left\{Z_{n}(h)-Z_{n}(0)\right\}_{h \in[0,1-t]}$, Relation (4.12) implies that $\{Z(t+h)-Z(t)\}_{h \in[0,1-t]} \stackrel{(d)}{=}\{Z(h)-Z(0)\}_{h \in[0,1-t]}$.

Acknowledgement. We would like to thank Professor Yves Meyer for his interest in our results and his careful reading of our article. His valuable advices greatly improved the original manuscript.

\section{References}

[1] P. Auscher, Wavelet bases for $L^{2}(R)$ with rational dilation factor, in: "Wavelets and their applications", Jones and Bartlett, Boston, MA, 1992, pp. 439-451.

[2] A. Ayache and J. LÉvy VÉHel, The generalized multifractional Brownian motion. 19th "Rencontres Franco-Belges de Statisticiens" 
(Marseille, 1998), Stat. Inference Stoch. Process. 3(1-2) (2000), $7-18$.

[3] A. Ayache And M. S. Taqqu, Rate optimality of wavelet series approximations of fractional Brownian motion, J. Fourier Anal. Appl. 9(5) (2003), 451-471.

[4] M. F. Barnsley, R. L. Devaney, B. B. Mandelbrot, H.-O. Peitgen, D. Saupe and R. F. Voss, "The science of fractal images", With contributions by Yuval Fisher and Michael McGuire, Springer-Verlag, New York, 1988.

[5] A. Benassi, S. Jaffard And D. Roux, Elliptic Gaussian random processes, Rev. Mat. Iberoamericana 13(1) (1997), 19-90.

[6] P. Doukhan, G. Oppenheim and M. S. Taqqu, eds., "Theory and applications of long-range dependence", Birkhäuser Boston, Inc., Boston, MA, 2003.

[7] S. Jaffard, Exposants de Hölder en des points donnés et coefficients d'ondelettes, C. R. Acad. Sci. Paris Sér. I Math. 308(4) (1989), 79-81.

[8] I. Karatzas and S. E. Shreve, "Brownian motion and stochastic calculus", Second edition, Graduate Texts in Mathematics 113, Springer-Verlag, New York, 1991.

[9] A. N. Kolmogorov, Wienersche Spiralen und einige andere interessante Kurven im Hilbertschen raum, Comptes Rendus (Doklady) de l'Académie des Sciences de l'URSS (N.S.) 26 (1940), 115-118.

[10] W. E. Leland, M. S. Taqqu, W. Willinger and D. V. WilSON, On the self-similar nature of Ethernet traffic (Extended version), IEEE/ACM Transactions on Networking 15 (1994), 1-15.

[11] M. A. Lifshits, "Gaussian random functions", Mathematics and its Applications 322, Kluwer Academic Publishers, Dordrecht, 1995.

[12] B. B. Mandelbrot and J. W. Van Ness, Fractional Brownian motions, fractional noises and applications, SIAM Rev. 10 (1968), $422-437$.

[13] Y. Meyer, F. Sellan and M. S. Taqqu, Wavelets, generalized white noise and fractional integration: the synthesis of fractional Brownian motion, J. Fourier Anal. Appl. 5(5) (1999), 465-494.

[14] G. C. Papanicolaou and K. Sølna, Wavelet based estimation of local Kolmogorov turbulence, in: "Theory and applications of long-range dependence", Birkhäuser Boston, Boston, MA, 2003, pp. $473-505$. 
[15] K. PARK AND W. Willinger, EDS., "Self-similar network traffic and performance evaluation", J. Wiley \& Sons, Inc., New York, 2000.

[16] R. F. Peltier And J. LÉvy VÉHel, Multifractional Brownian motion: definition and preliminary results, Technical Report 2645, Institut National de Recherche en Informatique et en Automatique, INRIA, Le Chesnay, France (1995).

Antoine Ayache:

UMR CNRS 8524

Laboratoire Paul Painlevé, Bat. M2, Université Lille 1, 59655 Villeneuve d'Ascq Cedex, France

and

UMR CNRS 8020

CLAREE, IAE de Lille,

104, Avenue du Peuple Belge 59034 Lille Cedex, France

E-mail address: Antoine.Ayache@math.univ-lille1.fr

Murad S. Taqqu:

Department of Mathematics and Statistics

Boston University

111 Cummington St.

Boston, MAS 02215

USA

E-mail address: murad@math.bu.edu

Rebut el 26 de gener de 2005 . 